\title{
A Novel Left Ventricular Volumes Prediction Method Based on Deep Learning Network in Cardiac MRI
}

\author{
Gongning Luo ${ }^{1}$, Guanxiong Sun ${ }^{1}$, Kuanquan Wang ${ }^{1}$, Suyu Dong ${ }^{1}$, Henggui Zhang ${ }^{1,2}$ \\ ${ }^{1}$ Harbin Institute of Technology, Harbin, China \\ ${ }^{2}$ University of Manchester, Manchester, UK
}

\begin{abstract}
Accurate estimation of left ventricle (LV) volumes plays an essential role in clinical diagnosis of cardiac diseases using MRI. Conventional methods of estimating ventricular volumes depend on the results of manual or automatic segmentation of MRI. However, manual segmentation of MRI sequences is extremely timeconsuming and subjective, and automatic segmentation is still a challenging task. Therefore, this study aims to develop a new $L V$ volumes prediction method without segmentation, motivated by deep learning technology and the large scale cardiac MRI (CMR) datasets from the second Annual Data Science Bowl (ADSB) in 2016. The experiments results shows that the predicted $L V$ volumes have high correlation with the ground truth. These results prove that the proposed method has big potential to be researched and applied in clinical diagnosis and screening of cardiac diseases.
\end{abstract}

\section{Introduction}

The cardiac disease is the leading killer of human being. Hence, the cardiac diseases get the more attention of researchers in recent years $[1,2]$. The left ventricle (LV) is the biggest chamber of heart, and the function indexes from LV have important indication significance for cardiac diseases, such as myocardial hypertrophy, myocardial ischemia, heart failure and so on. In recent years, more and more researches focus on the LV, especially in the field of computer-aided medicine [3-5]. The related LV function indexes, such as the end-diastole volumes (EDV), the end-systole volumes (ESV), and the eject fraction (EF), can be estimated from cardiovascular MR (CMR) images.

In the actually clinical diagnosis of cardiac diseases, the CMR is an important imaging modality and widely used because of its special advantages such as noninvasive detection, low radiation doses and high imaging quality [6]. Generally speaking, CMR is considered as gold standard in cardiac disease diagnosis. Hence, in this paper, the main modality of this research is CMR. Based on the CMR, the LV function estimation method is mainly classified into two kinds: the method based on LV segmentation and the method based on direct volumes prediction without segmentation. Generally, the current clinical diagnosis adopted manual or semi-automatic LV segmentation method. Though, the manual and semiautomatic methods are relative accurate compared to fully automatic LV segmentation methods. However, this task is time-consuming and tedious. Besides, the subjective variances exist during the processing of manual and semiautomatic segmentation. Hence, the fully automatic LV segmentation methods are still the research hot recently. However, the automatic LV segmentation is still an open and challenging task, due to characteristics of intensity level inhomogeneity and complex topological structures across different slices. Before 2011, the paper [7] reviewed the related LV segmentation algorithms detailedly. After 2011, the paper [8] reviews more methods in new views.

Compared with LV segmentation methods, the direct LV volumes prediction methods without segmentation are popular in recent years, especially along with the wide use of deep learning technology in the field of medical images processing $[9,10]$. The research group of Li Shuo proposed a series of methods for direct LV function indexes prediction method based on machine learning technologies [11-13], for example, the method based on adapted Bayesian formulation, the method based on linear support vector machine, and the method based on multiscale deep networks and regression forests.

In this paper, we conducted the new attempt in large scale CMR datasets of ADSB using the designed deep CNN networks. The rest of this paper is organized as follows. In the section 2, we introduced the basic frameworks about the proposed methods, including the data pre-processing and deep CNN design. In the section 3 , we showed the results of LV volumes prediction experiments. Finally, we concluded this paper in section 4 .

\section{Methods}

\subsection{Data Preprocessing}




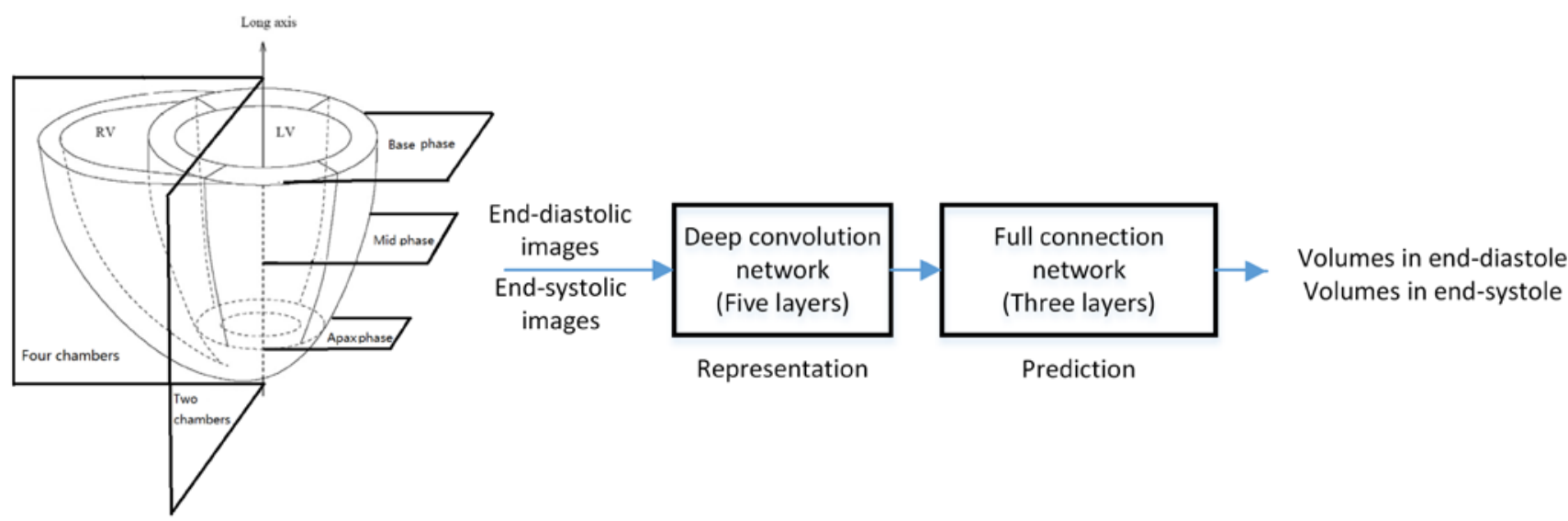

Figure 1. The pipeline of the proposed method

Actually, most of the previous works in the topic of LV volumes estimation are all attempted on small samples datasets (less than 100 subjects). What's more, these datasets are not open accessible and lack consistent measurement indexes, which limits the development of this research in some extent. Therefore, we conducted direct $\mathrm{LV}$ volumes prediction research using the new open accessible ADSB datasets. This datasets include 1140 subjects (more than 1026000 CMR images). To my best knowledge, ADSB are the biggest CMR images datasets up to now. However, this datasets contain subjects from different ages and different health levels. Therefore, before training and prediction using deep learning model, the pre-processing of CMR data is necessary.

We pre-processed the datasets in two aspects, the data selection and data features enhancement. In terms of the data selection, we just selected the subjects including long-axis and short-axis data, because the proposed framework is based on multi-views slices input, besides almost 5\% data lost the long-axis slices. As we can see in the Figure 1, one two chambers slice, one four chambers slice and three short-axis slices (apex, mid, and base slices) are the requirements for prediction networks.

In terms of image features enhancement, we adopted the Gabor filter to enhance the original CMR images. After a lot of experiments, we found that the images with Gabor filter have more substantial texture information, compared with the images without filter. The enhanced images and the original CMR images are all inputted into prediction networks.

\subsection{Deep CNN}

As we can see in the Figure 1, the proposed method is based on a deep learning framework, which includes a convolution network of five layers for features representation, and a full connection network of three layers for prediction. As described in above section, the two kinds of features (Gabor features and intensity) from multi-orientation (apex, mid, and base slices from short axis, four chambers slice and two chambers slice from long axis) images are used for initial inputs and the outputs of the network are the end-diastolic and endsystolic volumes (EDV, ESV). The deep learning network was trained and tested on cardiac MRI datasets from ADSB including 937 patients (600 patients for training model and 337 patients for testing).

In fact, the ADSB datasets provide us the ground truth of EDV and ESV, we integrated the EDV and ESV from 600 subjects into uniform training data. In other words, in the training step, we mixed the EDV and ESV to enhance the robustness of trained prediction model.

The prediction model contains eight layers, the five deep convolutional layers and the three full connection layers. The number of convolutional kernels are 4, 5, 6, 5, 5 in each layer respectively. We trained the convolutional networks based on the loss function in

$$
\text { Loss }=\sqrt{\frac{\sum_{i \in X}\left(X_{i}-Y_{i}\right)^{2}}{N}}
$$

where $\mathrm{N}$ refers to the amount of training examples, $\mathrm{X}_{\mathrm{i}}$ and $Y_{i}$ denote prediction value of the ith example and the true value of the ith example respectively.
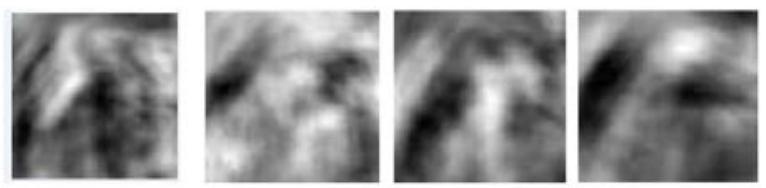

The feature maps in first convolutional layers
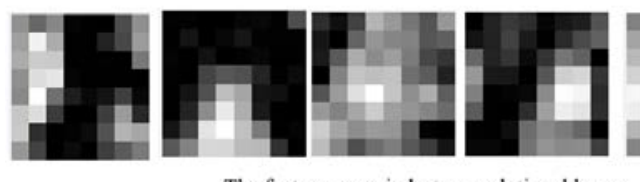

The feature maps in last convolutional layers

Figure 2. The features map from the model

Compared to the traditional hand-crafted features, the main advantage of deep learning is that it can learn some 
representational features automatically. This advantage is especially obvious in large scale datasets. The Figure 2 shows the example of the 4 feature maps in the first convolutional layers and the 5 feature maps in the last convolutional layers.

The model can be trained using back propagation algorithm (BP) and optimized using stochastic gradient descent (SGD). In this paper, the training is conducted for 300 iterations. We selected the model at the minimum loss value of validation datasets (the 600 training data include 400 training subjects and 200 validation subjects) during the training process as the finial optimal prediction model. This data distribution method can enhance the generalization ability of the final prediction model to some extent.

\section{Results and discussion}

\section{1. $\quad$ Metrics}

The proposed method was evaluated by the recognized criterion in this field, including the linear regression fit (y $=\mathrm{ax}+\mathrm{b}$, ideally $\mathrm{a}=1$ and $\mathrm{b}=0$ ), correlation coefficient (R) and mean errors (ME) \pm the standard deviation (SD) of absolute errors between the predicted values and the true values for EDV, ESV and ejection fraction (EF).

\subsection{Environment of experiments}

We conducted the experiments in the workstation, whose configurations are $3.4 \mathrm{GHz}$ Core i7 CPU, 64GB RAM, Nvidia TiTan X (12GB memories). The codes are based on python language and Keras deep learning framework. The SGD learning ratio is 0.0001, the batch size is 64 in per iteration of training, the number of iteration is 300 for training a $\mathrm{LV}$ volumes prediction model, and we adopted the Gaussian weight initializations to set the initial networks.

\subsection{Prediction accuracy}

The clinical indexes predicted by our method were compared with ground truth from ADSB. Note that the tested data in this paper are selected from validated data of ADSB, because some data loss exists in the original datasets. Finally, 337 subjects are chosen to test the prediction model. As shown in the Figure 3, the results are as following: EDV: $\mathrm{y}=0.91 \mathrm{x}+11.7, \mathrm{R}=0.95$, $\mathrm{ME}=5.1 \pm 3.1 \mathrm{ml} ; \mathrm{ESV}: \mathrm{y}=0.97 \mathrm{x}+9.5, \mathrm{R}=0.92, \mathrm{ME}=3.6 \pm$ 2.7ml; $\mathrm{EF}: \mathrm{y}=0.87 \mathrm{x}+0.2, \mathrm{R}=0.9, \mathrm{ME}=6.1 \pm 4.1 \%$. Compared to the ESV, the accuracy of prediction on EDV is lower. This situation is contrary to the segmentation problems, in which the EDV is more accurate than ESV. The reason may be the relatively bigger values range of EDV, which results in higher solution error. In the future, the independent model for EDV prediction and ESV prediction should be explored for balance of the accuracy between EDV and ESV. Additionally, in some cases, EDV is lower than ESV in the same subject. To address this problem, some judgment principles should be added to the direct prediction model.
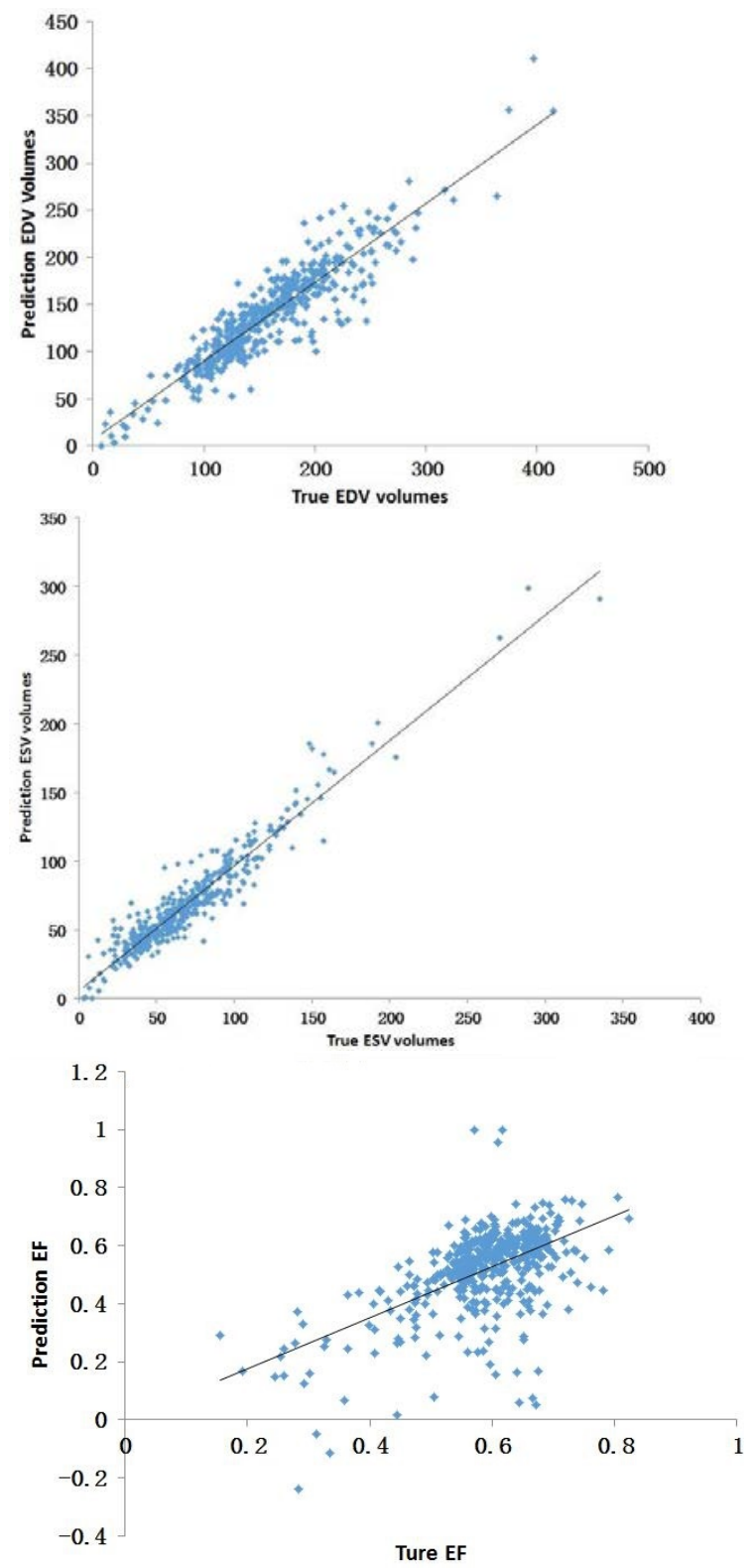

Figure 3. The correlation analysis of prediction results for EDV, ESV, and EF.

\subsection{Efficiency}

We report the efficiency of the proposed method in terms of the number of CNN layers and model training.

- In the term of CNN networks, the number of 
CNN layers is the leading factor for computation cost. After a lot of attempts with different depth of the CNN, we found the five CNN layers are good balance between computation efficiency and location accuracy. Some experiments results also show that the too many CNN layers with deeper networks may results in over fitting problems.

- In the term of training efficiency, the designed deep CNN can achieve 2s in every iteration of training. Besides, as shown in Figure 4, the proposed model has fast convergence rate especially at the start of training. The power fitting of the trend of loss decrease also is shown in Figure 4, which shows the good reduce trend of the proposed prediction model.

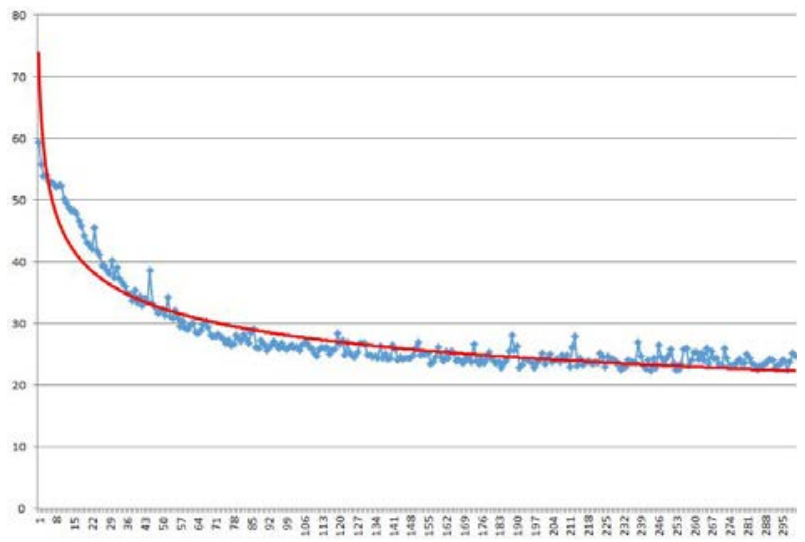

Figure 4 . The trend of loss decrease during training

\section{Conclusion}

In the paper, we proposed an end-to-end framework to address the LV volumes prediction problem. Though some limitations exist, the experiments results show that the proposed method has bigger potential to be further improved and applied in the clinical diagnosis. In the future, we will try to balance of accuracy between the EDV and ESV, as well as the prediction accuracy of EF.

\section{Acknowledgements}

This work is supported by the National Natural Science Foundation of China (NSFC) under Grant No. 61571165 and No. 61572152. The Titan X used for this research was donated by the NVIDIA Corporation. Thanks for Annual Data Science Bowl in 2016 (ADSB), who provided the CMR data for this research work.

\section{References}

[1] Selvanayagam JB. Non-Invasive Cardiac Imaging: Past, Present and Future. Heart, lung \& circulation. 2016;25(8):755-6.
[2] Luo GN, An R, Wang KQ, Dong SY, Zhang HG. A deep learning network for right ventricle segmentation in shortaxis MRI. Computing in Cardiology 2016;43.

[3] Avendi MR, Kheradvar A, Jafarkhani H. A combined deeplearning and deformable-model approach to fully automatic segmentation of the left ventricle in cardiac MRI. Med Image Anal. 2016;30:108-19.

[4] Dong SY, Luo GN, Sun GX, Wang KQ, Zhang HG. A left ventricular segmentation method on $3 \mathrm{D}$ echocardiography using deep learning and snake. Computing in Cardiology: IEEE; 2016.

[5] Dong SY, Luo GN, Sun GX, Wang KQ, Zhang HG. A combined multi-scale deep learning and random forests approach for direct left ventricular volumes estimation in 3D echocardiography. Computing in Cardiology 2016;43.

[6] Suinesiaputra A, Medrano-Gracia P, Cowan BR, Young AA. Big Heart Data: Advancing Health Informatics Through Data Sharing in Cardiovascular Imaging. Ieee J Biomed Health. 2015;19(4):1283-90.

[7] Petitjean C, Dacher JN. A review of segmentation methods in short axis cardiac MR images. Medical Image Analysis. 2011;15(2):169-84.

[8] Peng P, Lekadir K, Gooya A, Shao L, Petersen SE, Frangi AF. A review of heart chamber segmentation for structural and functional analysis using cardiac magnetic resonance imaging. Magma. 2016;29(2):155-95.

[9] Shin HC, Roth HR, Gao M, Lu L, Xu Z, Nogues I, et al. Deep Convolutional Neural Networks for Computer-Aided Detection: CNN Architectures, Dataset Characteristics and Transfer Learning. IEEE Trans Med Imaging. 2016;35(5):1285-98.

[10] Zhang L, Yang F, Zhang YD, Zhu YJ. Road crack detection using deep convolutional neural network. IEEE International Conference on Image Processing (ICIP 2016)2016.

[11] Wang Z, Salah MB, Gu B, Islam A, Goela A, Li S. Direct estimation of cardiac biventricular volumes with an adapted Bayesian formulation. IEEE transactions on bio-medical engineering. 2014;61(4):1251-60.

[12] Afshin M, Ben Ayed I, Punithakumar K, Law M, Islam A, Goela A, et al. Regional assessment of cardiac left ventricular myocardial function via MRI statistical features. IEEE Trans Med Imaging. 2014;33(2):481-94.

[13] Zhen X, Wang Z, Islam A, Bhaduri M, Chan I, Li S. Multiscale deep networks and regression forests for direct biventricular volume estimation. Med Image Anal. 2016;30:120-9.

Address for correspondence.

Kuanquan Wang

Mailbox 332, Harbin Institute of Technology

Harbin 150001, China

wangkq@hit.edu.cn 